\title{
Targeting CAG repeat RNAs reduces Huntington's disease phenotype independently of huntingtin levels
}

\author{
Laura Rué, ${ }^{1,2,3}$ Mónica Bañez-Coronel,, 4,5,6,7 Jordi Creus-Muncunill, ${ }^{1,2,3}$ Albert Giralt, ${ }^{1,2,3}$ Rafael Alcalá-Vida, ,2,3 Gartze Mentxaka, ${ }^{4,5,6,7}$ \\ Birgit Kagerbauer, ${ }^{4,5,6,7}$ M. Teresa Zomeño-Abellán, ${ }^{4,5,6,7}$ Zeus Aranda, ${ }^{4,5,6,7}$ Veronica Venturi, ${ }^{4,5,6,7}$ Esther Pérez-Navarro, ${ }^{1,2,3}$ \\ Xavier Estivill, 4,5,6,7 and Eulàlia Martii ${ }^{4,5,6,7}$

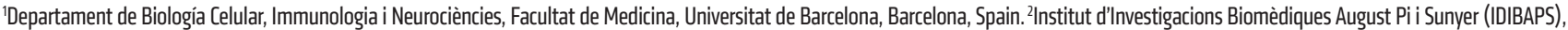 \\ Barcelona, Spain. ${ }^{3}$ Centro de Investigación Biomédica en Red sobre Enfermedades Neurodegenerativas (CIBERNED), Madrid, Spain. ${ }^{4}$ Cenomics and Disease Group, Centre for Genomic Regulation (CRG), \\ The Barcelona Institute for Science and Technology, Barcelona, Spain. '5niversitat Pompeu Fabra (UPF), Barcelona, Spain. ${ }^{6}$ Centro de Investigación Biomédica en Red Epidemiología y Salud Pública \\ (CIBERESP), Barcelona, Spain. ${ }^{7}$ Hospital del Mar Research Institute (IMIM), Barcelona, Catalonia, Spain.
}

Huntington's disease (HD) is a polyglutamine disorder caused by a CAG expansion in the Huntingtin (HTT) gene exon 1. This expansion encodes a mutant protein whose abnormal function is traditionally associated with HD pathogenesis; however, recent evidence has also linked HD pathogenesis to RNA stable hairpins formed by the mutant $H T T$ expansion. Here, we have shown that a locked nucleic acid-modified antisense oligonucleotide complementary to the CAG repeat (LNA-CTC) preferentially binds to mutant HTT without affecting HTT mRNA or protein levels. LNA-CTCs produced rapid and sustained improvement of motor deficits in an R6/2 mouse HD model that was paralleled by persistent binding of LNA-CTC to the expanded HTT exon 1 transgene. Motor improvement was accompanied by a pronounced recovery in the levels of several striatal neuronal markers severely impaired in R6/2 mice. Furthermore, in R6/2 mice, LNA-CTC blocked several pathogenic mechanisms caused by expanded CAG RNA, including small RNA toxicity and decreased $R n 45 s$ expression levels. These results suggest that LNA-CTCs promote neuroprotection by blocking the detrimental activity of CAG repeats within HTT mRNA. The present data emphasize the relevance of expanded CAC RNA to HD pathogenesis, indicate that inhibition of $H T T$ expression is not required to reverse motor deficits, and further suggest a therapeutic potential for LNA-CTC in polyglutamine disorders.

\section{Introduction}

Huntington's disease (HD) is an incurable neurodegenerative disease caused by more than 36 CAG repeats within the HTT gene (1). In HD and other polyglutamine disorders, CAG repeat expansion leads to an elongated polyglutamine fragment in the encoded protein. Pathogenesis has been traditionally linked to abnormal function of the mutant protein that, through misfolding and aggregation, promotes aberrant interactions with other proteins (2-4). The mutant protein promotes proteome disruption and alterations in neurotransmitter release, mitochondrial function, and transcriptional activity, among other mechanisms (4).

The most recent advances in understanding HD pathogenesis have revealed the coexistence of mutant protein toxicity and detrimental activity of mutant/expanded CAG RNAs (5-9). Long CAG-trinucleotide repeats form RNA stable hairpin structures (10), with the stem portion presenting protein-binding properties (8, 11). Abnormal interaction of specific proteins with expanded CAG repeats results in alterations of the normal function of these proteins and consequent perturbations in gene expression and alternative splicing $(8,12)$. In addition, hairpin-like structures in mutant/

Authorship note: L. Rué and M. Bañez-Coronel contributed equally to this work and are co-first authors.

Conflict of interest: The authors have declared that no conflict of interest exists. Submitted: June 9, 2015; Accepted: September 8, 2016.

Reference information: J Clin Invest. 2016;126(11):4319-4330. doi:10.1172/JCI83185. expanded transcripts undergo repeat-associated non-ATG (RAN) translation (13), which adds complexity to our understanding of the pathogenic processes in trinucleotide repeat expansion diseases.

An important question that has not been thoroughly addressed in $\mathrm{HD}$ and other polyglutamine diseases is the relative contribution of the full-length protein and/or the RNA-dependent mechanisms underlying disease pathogenesis. The elucidation of this question has potentially important translational implications.

Blocking or inhibiting the mutant HTT allele is an enticing therapeutic strategy, and several types of modified antisense oligonucleotides (ASOs) targeting the CAG tract take advantage of differences in the CAG repeat size between the normal and expanded alleles to selectively inhibit mutant HTT expression $(14,15)$. Locked nucleic acid-modified (LNA-modified) ASOs (LNA-ASOs) complementary to the CAG repeats have been found to block RNA translation to a variable extent, depending on the type of LNA-ASO (16-18). We have previously shown that a particular type of LNA-ASO with CTG repeats (LNA-CTG) abrogates the toxicity produced by CAG repeat small RNAs (sRNAs) generated from mutant HTT transcripts in a human neuronal cell model (9). However, the effect of LNACTGs in vivo has not been examined.

Here, we have characterized the effect of LNA-CTGs on HTT expression and assessed whether their injection into the striatum of the R6/2 mouse model of HD affects motor symptoms. Our data show that LNA-CTGs dramatically improve motor deficits in 
A

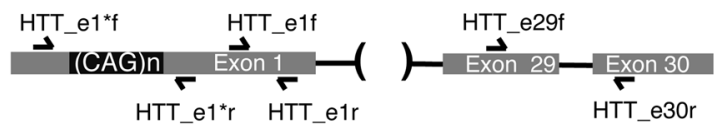

B
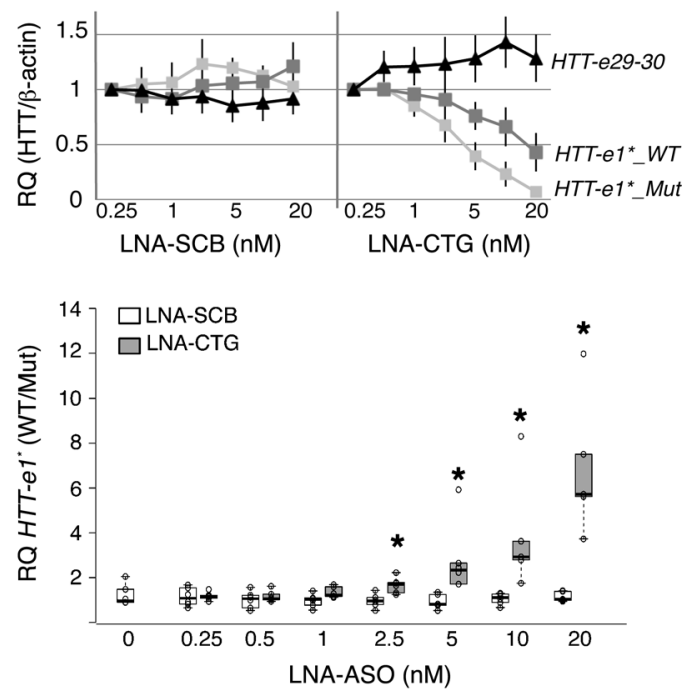

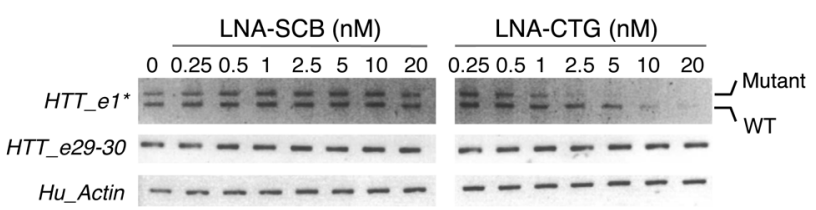

C

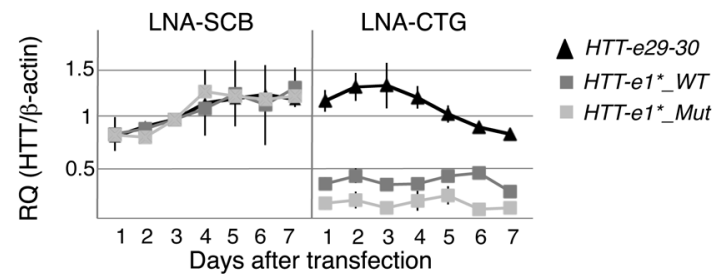

Figure 1. LNA-CTG preferentially binds to expanded HTT mRNA in HD fibroblasts, without inhibiting HTT protein levels. (A) Scheme showing the binding sites of the primers used for PCR amplification in HTT exon 1 (HTT_e1* and HTT_e1 sets of primers) and HTT exons $29-30$ (HTT_e29-30 set of primers). The black line represents introns. (B) HTT expression in HD fibroblasts (44_CAC repeats) transfected with different concentrations of LNA-CTG or LNA-SCB, 48 hours after transfection. Graph shows the RQ of HTT using the primer set HTT-e ${ }^{*}$ detecting WT (HTT-e ${ }^{*}-$ WT) or mutant (HTT-e $1^{*}$-Mut) HTT-exon 1 and the primer set HTT_e29-30 detecting HTT-e29-30. Densitometric determinations were normalized to the $\beta$-actin PCR product and referred to the mock-transfected condition with a value of 1 . Results are expressed as the mean \pm SEM ( $n=6$ ). Box plot shows quartile values of the RQ of WT versus mutant HTT-e1* mRNA levels normalized to $\beta$-actin levels in HD fibroblasts transfected with LNA-CTC or LNA-SCB. A representative gel electrophoresis of the PCR products is shown. ${ }^{*} P<0.05$, by Mann-Whitney $U$ test with Bonferroni's correction for multiple comparisons ( $n=6$ independent transfections). (C) HTT expression at different time points after transfection with 15 nM LNA-ASOs in HD fibroblasts (68_CAG repeats). The mean determinations \pm SEM of HTT-e1*-WT and HTT-e1*-Mut and HTT-e29-30 relative to $\beta$-actin PCR products and a representative PCR gel electrophoresis are shown $(n=3)$. (D) Dot plots show Western blot densitometric analysis of WT HTT (WT-HTT) versus mutant HTT (mHTT) protein levels and total HTT (WT and mutant alleles) protein levels normalized to $\beta$-actin and expressed relative to an LNA-SCB-transfected sample with a value of 1 . A representative immunoblot is shown $(n=3)$.

R6/2 mice and block RNA-associated detrimental pathways, without significantly affecting mutant $H T T$ levels. The present results pose a challenging question about the type of molecular mechanisms underlying HD pathogenesis.

\section{Results}

To characterize the effects of LNA-CTGs in human full-length $H T T$, we transfected either LNA-CTG or the analogous scrambled control LNA-ASO (LNA-SCB) into HD fibroblasts and monitored LNA-CTG binding to the CAG repeat as well as HTT expression levels (mRNA and protein) (Figure 1). LNA-CTG binding to HTT mRNA was determined by the lack of PCR amplification within the LNA-bound region due to the strong incompatibility of LNA-CTG:CAG duplexes with retrotranscription and subsequent PCR amplification (Supplemental Figure 1; supplemental material available online with this article; doi:10.1172/JCI83185DS1).
Increasing LNA-CTG concentrations resulted in progressively decreased detection of both the mutant (e1_Mut) and WT (e1_WT) alleles by PCR when using the HTT_e1* set of primers, which span the CAG repeat within the HTT exon 1 transgene (HTT-e1) (Figure 1A and Methods). The blockage of HTT-e1 amplification preferentially affected the mutant allele, producing a 6 -fold reduction at the highest LNA-CTG dose (20 nM) (Figure 1B). Quantitative PCR (qPCR) with primers outside the CAG repeat revealed no changes in HTT mRNA levels. In addition, $H T T$ transcripts and protein levels were not altered at 15-nM doses of LNA-CTG, which strongly inhibited HTT-e1 amplification for up to 7 days after transfection of LNA-CTG (Figure 1, C and D), suggesting that LNA-CTG binding prevents CAG repeat availability but does not result in RNA or protein degradation. These results are consistent with previous observations showing a weak inhibition of mutant HTT protein 
A

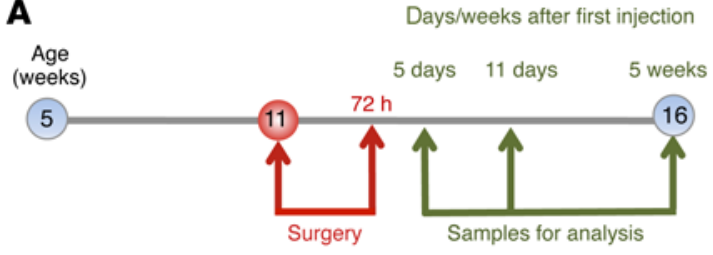

C

D

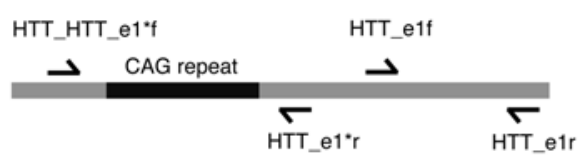

5 days after injection

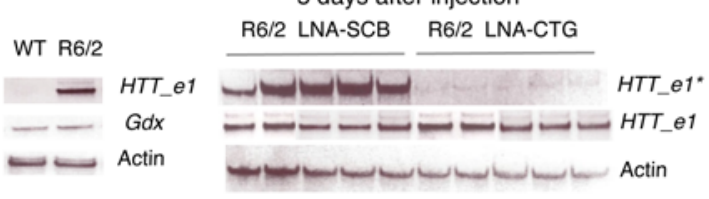

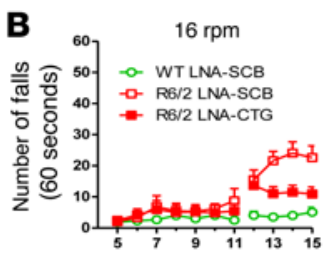
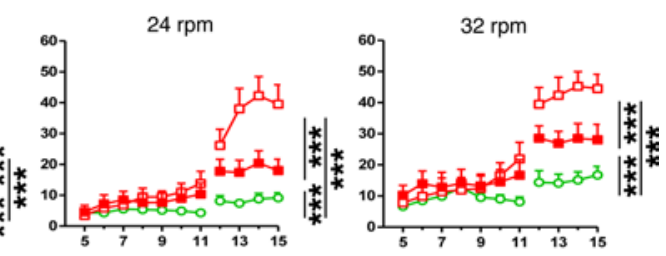

HTT
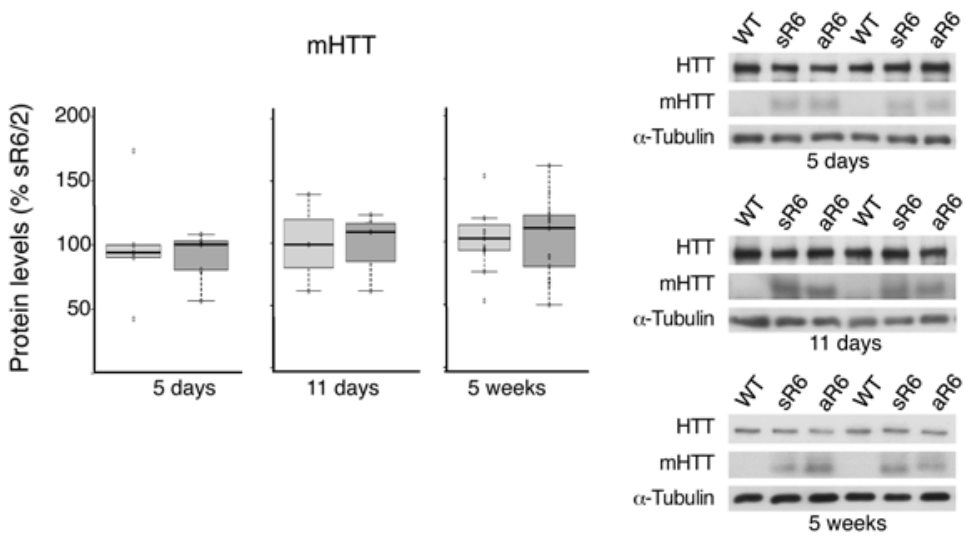

5 days after injection

5 days after injection
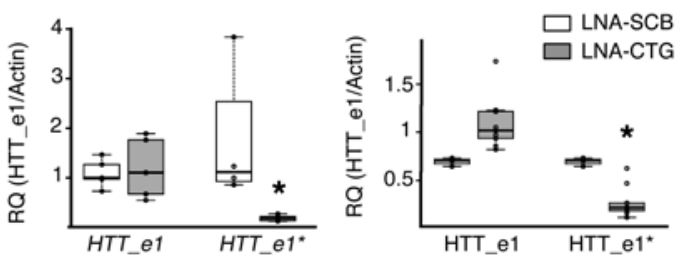

Figure 2. Intrastriatal injection of LNA-CTC induces rapid motor improvement in the R6/2 mouse model of HD, without affecting WT or mutant HTT expression levels. (A) Schematic representation of pharmacological treatment administered and analyses. (B) Results from the rotarod test performed at 16,24 , and $32 \mathrm{rpm}$ by mice between 5 and 15 weeks of age. Values represent the number of falls within 60 seconds as the mean $\pm S E M$ (WT $n=16$; R6/2 $n=23$; after surgery: WT $n=11 ;$ R6/2 LNA-SCB $n=11 ;$ R6/2 LNA-CTC $n=12)$. ${ }^{* *} P<0.001$ versus WT vehicle-treated mice, as determined by 2-way ANOVA with Bonferroni's post-hoc correction. (C) WT HTT (WT-HTT) and mutant HTT-e1 (mHTT) protein levels in the striatum of WT and R6/2 mice following intrastriatal injection of LNA-SCB or LNA-CTC, as analyzed by Western blotting. Box plots show the percentage of HTT and mHTT in the striatum of WT and/or R6/2 mice at different time points after LNA-ASO injection. Densitometric HTT and mHTT determinations were normalized using $\alpha$-tubulin as an endogenous control and expressed as a percentage of a WT or R6/2 sample. Representative immunoblots are shown. WT: LNA-SCB-injected WT mice; sR6: LNA-SCB-injected R6/2 mice; aR6/2: LNA-CTG-injected R6/2 mice. Data were analyzed with Kruskal-Wallis (WT-HTT) and Mann-Whitney $U$ (mHTT) tests $(n=4-12$ animals per group). (D) HTT-e1 RNA transgene expression was analyzed by RT-PCR using the primer sets HTT_e1* or HTT_e1. Representative PCR products from animals injected with LNA-CTC or LNA-SCB are shown. $\beta$-Actin and $G d x$ amplification was used for internal controls. Box plots show RQ (obtained by densitometry) of HTT-e1 PCR products normalized to $\beta$-actin levels and referred to a control R6/2 LNA-SCB sample with a value of 1 . ${ }^{*} P<0.01$, when comparing LNA-SCB versus LNA-CTG-injected R6/2 mice in HTT_e ${ }^{*}$ PCR amplifications; Mann-Whitney $U$ test with Bonferroni's correction ( $n=5-8$ mice per group).

expression by some types of LNA-ASOs complementary to the CAG repeats, when used at similar concentrations (17).

At the 15-nM dose, we found reduced nuclear foci in HD fibroblasts when using a fluorescent ISH probe targeting the CAG repeats. This result provides additional evidence for the direct binding between LNA-CTG and the mutant $H T T$ transcript (Supplemental Figure 2).

Additional experiments using a human neuronal cell model expressing HTT-e1 confirmed a preferential binding of LNA-CTG for expanded HTT-e1 RNA (Supplemental Figure 3), while protein levels remained unaffected by LNA-CTG administration (Supplemental Figure 4A). Collectively, these data indicate that LNACTG can be used at concentrations that show efficient and pref- erential binding to the CAG repeats in expanded HTT mRNA and that this binding is compatible with HTT translation. Thus, LNACTGs are an optimal tool for dissecting the effects of expanded CAG repeat blockage that are not necessarily linked to inhibition of HTT expression.

To evaluate the effect of LNA-CTG on HD in vivo, we used the R6/2 HD mouse model that expresses a human HTT-e1 with 90 CAG repeats. WT and R6/2 mice were assessed with the rotarod task from 5 weeks of age to evaluate motor coordination (Supplemental Figure 5A). The mice received 2 consecutive intrastriatal injections of LNA-CTG or control LNA-SCB at 11 weeks of age, when motor symptoms appeared in the R6/2 mice (Figure 2A). Deficits in motor coordination in the R6/2 mice were 
Table 1. Top dysregulated genes in the microarray analysis that were validated by qPCR

\begin{tabular}{|c|c|c|c|c|c|c|c|c|}
\hline \multirow[b]{2}{*}{ Gene name } & \multirow[b]{2}{*}{ Systematic name } & \multicolumn{2}{|c|}{ Microarrays } & \multicolumn{2}{|c|}{ qPCR (reference gene: $\mathrm{Hprt}$ ) } & \multicolumn{2}{|c|}{ qPCR (reference gene: Tbp) } & \multirow[b]{2}{*}{$\begin{array}{l}>4 \text { CAG in the } \\
\text { array probe }\end{array}$} \\
\hline & & Fold change & Adjusted $P$ value & Fold change & $P$ value & Fold change & $P$ value & \\
\hline Arhgap17 & NM_144529 & 0.02 & 0.00 & 0.02 & 0.00 & 0.13 & 0.00 & Yes \\
\hline Crebbp & NM_001025432 & 0.03 & 0.00 & 0.04 & 0.00 & 0.05 & 0.00 & Yes \\
\hline $\operatorname{Trp63}$ & NM_001127261 & 0.06 & 0.00 & 0.07 & 0.00 & 0.08 & 0.00 & Yes \\
\hline Pf4 & NM_019932 & 0.12 & 0.10 & 0.26 & 0.00 & 0.29 & 0.00 & No \\
\hline Mdh2 & NM_008617 & 0.14 & 0.01 & 0.09 & 0.00 & 0.11 & 0.00 & Yes \\
\hline Phlpp1 ${ }^{\mathrm{A}}$ & NM_133821 & 2.33 & 0.16 & 0.94 & 0.56 & - & - & No \\
\hline$P 2 r y 13^{A}$ & NM_028808 & 2.62 & 0.05 & 1.04 & 0.65 & 1.18 & 0.07 & No \\
\hline Pde3b & NM_011055 & 2.70 & 0.12 & 1.33 & 0.04 & 1.50 & 0.00 & No \\
\hline
\end{tabular}

Two quantifications were performed using Hprt or Tbp as reference genes. The fold change is shown for R6/2 mice injected with LNA-CTC versus R6/2 mice injected with LNA-SCB. The last column indicates if the probe in the microarray contained at least 4 consecutive CAG repeats. ${ }^{A}$ Cene was not validated by $q P C R$.

significantly attenuated as soon as 1 week after the first intrastriatal injection of LNA-CTG (i.e., at 12 weeks of age) and persisted for at least 4 additional weeks (i.e., 15 weeks of age) (Figure 2B). Injection of LNA-CTG did not produce significant changes in BW or muscular strength (Supplemental Figure 5, B and C). In contrast, WT animals treated with LNA-CTG showed no alterations in their performance on the rotarod compared with WT mice injected with LNA-SCB (Supplemental Figure 6A), suggesting that LNA-CTG specifically improved motor coordination performance in $\mathrm{R} 6 / 2$ mice.

Motor improvement was not linked to any changes in the expression of the HTT-e1 transgene or endogenous (HTT) proteins or HTT-e1 mRNA at different time points after LNA-CTG administration (Figure 2, C and D, Supplemental Figure 6B, and Supplemental Figures 7-9). The lack of effect on HTT-e1 and endogenous HTT protein was demonstrated with MAB2166, EM48, and MAB5492 antibodies (Figure 2 and Supplemental Figure 8). However, the lack of HTT-e1 mRNA amplification with primers spanning the CAG repeat suggests LNA-CTG binding to the expanded transgene (Figure 2D and Supplemental Figure 9), as shown in HD fibroblasts (Figure 1). Similar results were observed in a mouse full-length $\mathrm{Htt}$ context upon transfection of LNA-CTG into immortalized striatal neuronal cells expressing endogenous levels of normal and expanded HTT protein with 7 (STHdh ${ }^{\mathrm{Q} 7 / \mathrm{Q}^{7}}$ ) and 111 (STHdh ${ }^{\mathrm{Q} 11 / \mathrm{Q}^{111}}$ ) glutamines, respectively (19) (Supplemental Figure 10).

To identify pathways underlying the beneficial effects of LNA-CTG, we examined overall gene expression changes in striatal samples shortly before motor improvement was evident, e.g., 5 days after the first injection. We detected a total of 56 dysregulated genes $(n=5-7$ animals per group; adjusted $P<0.2$, fold change greater than or less than 1.2) in LNA-CTG- versus LNASCB-treated R6/2 mice (Table 1, Supplemental Table 1, and Sup- plemental Figure 11). Among the microarray probes that detected strong downregulation, we observed a significant enrichment in those that contained at least 4 consecutive CAG repeats (Table 1). For these genes, qPCR validation suggested that downregulation probably reflected an interference of LNA-CTG with retrotranscription during the process of sample preparation for microarray hybridization, rather than a true decrease in expression (Figure 3 and Table 1). Additional genes containing a short CAG repeat stretch (Maml2, Mef2a, Nr3c1, and Satb1) that were not specifically targeted by the probe in the microarray also showed an apparent downregulation when primers spanning the CAG repeat were used; however, this trend was not confirmed when primers outside the CAG repeat were used (Supplemental Figure 12A). In addition, no modifications in the corresponding protein levels were detected after treatment with LNA-CTG (Supplemental Figure 12B), suggesting that binding of LNA-CTG to endogenously expressed short CAG repeats was compatible with protein translation, as shown for the HTT gene (Figures 1 and 2).

LNA-CTG similarly bound to genes harboring short CAG repeats in WT mice striatum, without noticeable effects on the transcript levels (Supplemental Figure 13). The lack of effect of LNA-CTG on WT motor coordination and the rapid and sustained beneficial effects of LNA-CTG on R6/2 mice suggest that off-target binding to endogenously expressed short CAG repeats had no detrimental functional consequences at the tested dose.

In addition, we examined the protein levels of several neuronal markers whose expression is strongly decreased in striatal cells expressing mutant HTT (20-25) (Figure 4). Treatment with LNACTG resulted in a significant recovery of protein levels of the $\mathrm{PH}$ domain and leucine-rich repeat protein phosphatase 1 (PHLPP1), dopamine- and cyclic AMP-regulated phosphoprotein of $32 \mathrm{kDa}$ (DARPP-32), striatal-enriched protein tyrosine phosphatase 46 (STEP46), and postsynaptic density protein 95 (PSD95). A simi- 
A

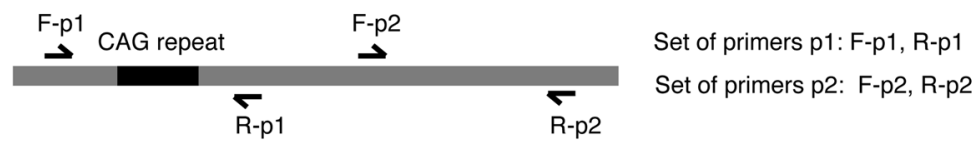

B
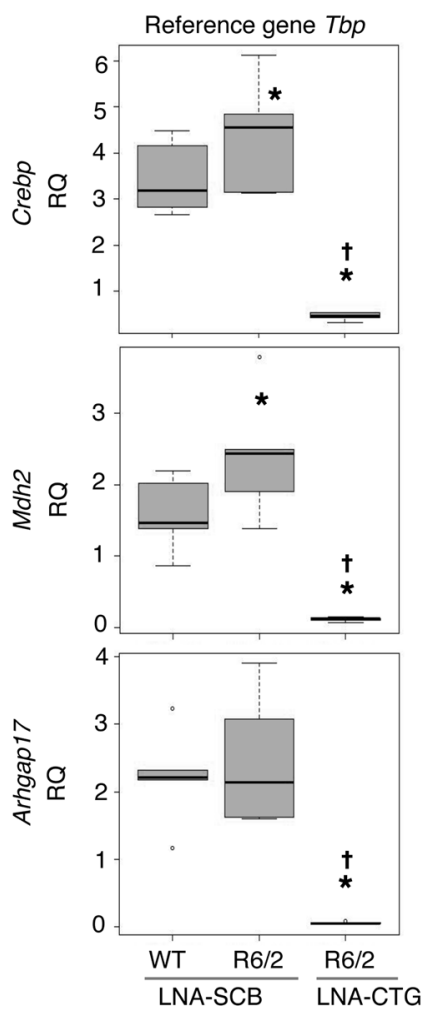

mRNA levels, set of primers $\mathrm{p} 1$
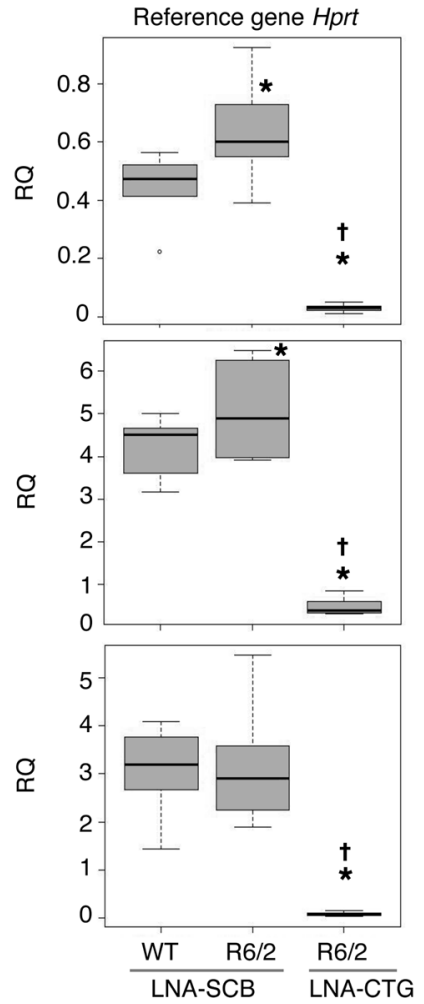

mRNA levels, set of primers p2
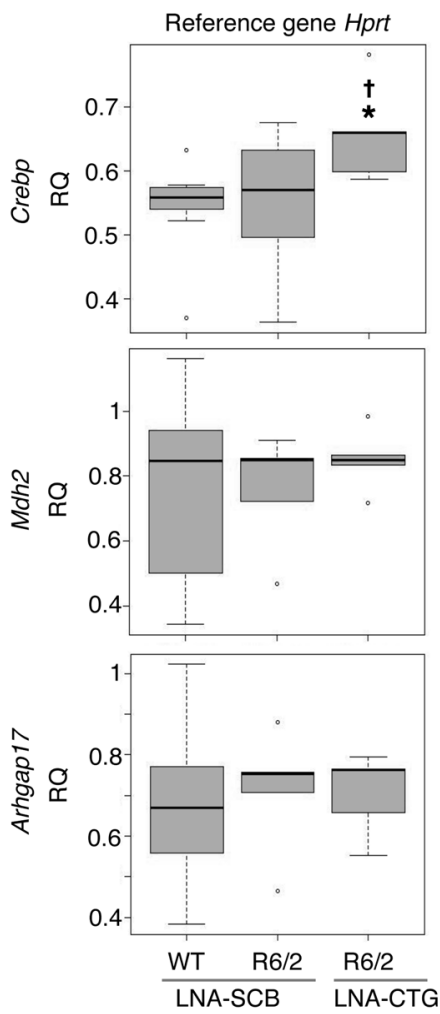

Figure 3. Downregulation of specific genes containing CAG repeats, detected by microarray analysis, reflects binding of LNA-CTC to the CAG stretch. (A) Schema showing the binding site of the primers spanning the CAG repeats (p1) and primers mapping outside the CAG repeats (p2). (B) The expression levels of Crebbp, Mdh2, and Arhgap17 are shown in WT animals injected with LNA-SCB and R6/2 mice injected with LNA-SCB or LNA-CTC. A WT sample was used as a reference for RQ. Quantification was normalized to Tbp or Hprt as independent reference genes. ${ }^{*} P<0.01$ with respect to WT LNA-SCB mice; ${ }^{t} P<0.01$ with respect to R6/2 LNA-SCB mice. Data were analyzed using a linear mixed-effects model and Bonferroni's correction was applied for multiple comparisons ( $n=5-7$ mice per group).

lar tendency was observed for CREB-binding protein (CBP) and enkephalin (ENK) (Figure 4A). The trend toward the recovery of CBP protein levels and the binding of LNA-CTG to the CAG repeats in the Crebbp transcript (Figure 3B) further reinforce the idea that LNA-CTG binding to CAG repeats does not hamper translation of the targeted transcript. Parallel changes in the transcripts encoding for DARPP-32, ENK, STEP, and PSD95 were not detected according to the microarray analysis, indicating that LNA-CTGs posttranscriptionally modulate the expression of these genes. LNA-CTG did not alter the levels of these striatal markers in WT mice (Supplemental Figure 6B), which is in line with the specific effect of LNA-CTG in R6/2 mice. Immunofluorescence analysis of DARPP-32-positive neurons confirmed the Western blot results and suggested that DARPP-32 downregulation in striatal neurons was reversed close to the injection site (Figure $4 \mathrm{~B}$ ).

Since LNA-CTGs bind to the expanded CAG repeats in mutant $H T T-e 1$ RNA, we evaluated whether pathogenic mechanisms directly dependent on expanded RNA were modified in R6/2 mice receiving LNA-CTG. Dicer activity on the hairpin-like double-stranded structure in expanded CAG repeats of HTT-e1 results in the biogenesis of small CAG-repeated RNAs (sCAGs) (26) with toxic activity (9). In addition, binding of dicer to expanded CAG repeats has been proposed to contribute to abnormal miR expression profiles that may underlie pathogenic alterations in gene expression (27).

To evaluate whether the detrimental effect of sRNAs was reversed by LNA-CTG, we isolated an sRNA-enriched fraction (<200 nt) from the striatum of WT and R6/2 mice injected with LNA-SCB and from R6/2 mice injected with LNA-CTG (pooled extracts from at least 11 mice per condition) and determined cell death after transfection into a differentiated neuronal cell line (Figure 5A). The sRNA fraction isolated from R6/2 mice injected with LNA-SCB produced a significant increase in cell death that was not detected upon transfection of sRNAs isolated from R6/2 mice injected with LNA-CTG. This result suggests that LNA-CTGs block the sRNA pathogenic mechanisms in the mouse brain. 

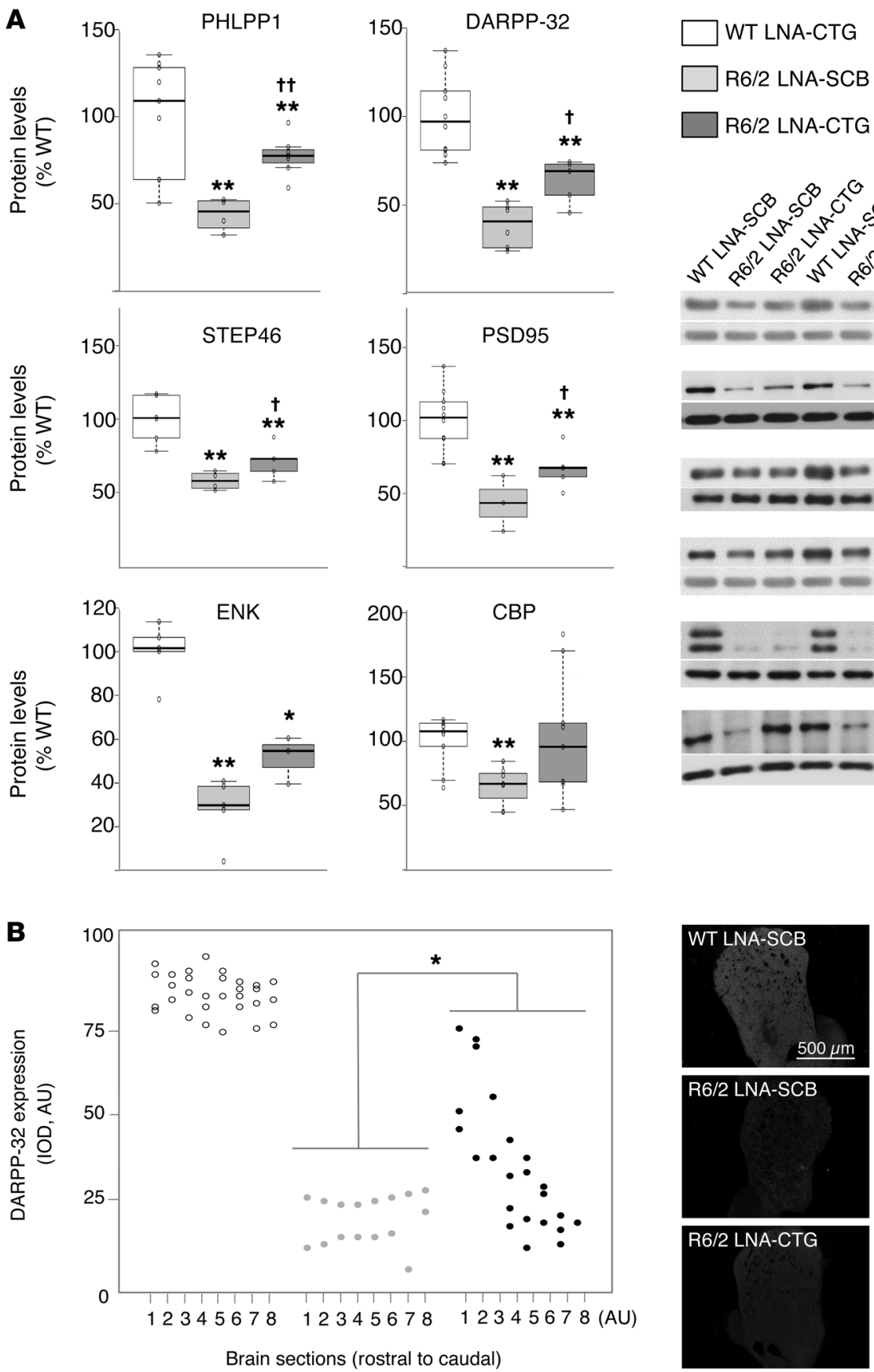

Figure 4. Intrastriatal injection of LNA-CTC recovers protein levels of several striatal markers in R6/2 mice. (A) Levels of specific neuronal proteins 5 days after the first intrastriatal injection of LNA-SCB into WT and R6/2 mice and LNA-CTC into R6/2 mice. Box plots represent the densitometric protein quantification normalized to actin or tubulin and expressed relative to WT mice. Representative immunoblots are shown. Data were analyzed using the Kruskal-Wallis test, providing significant differences in all cases $(P<0.05)$. To determine statistical post-hoc differences between pairs of groups, the Mann-Whitney $U$ test with Bonferroni's correction was used $(n=6-8) .{ }^{*} P<0.05$ and ${ }^{* *} P<0.01$, compared with LNA-SCB-injected WT mice; ${ }^{\dagger} P<0.05$ and ${ }^{\dagger+} P<0.01$, compared with LNA-SCB-injected R6/2 mice. (B) Individual points show the fluorescence intensity of DARPP-32 staining along rostral-to-caudal striatal sections in WT LNA-SCB, R6/2 LNA-SCB, and R6/2 LNA-CTC mice 5 days after the first intrastriatal injection. IOD, integrated optical density. ANOVA was applied using a correlation structure for the repeated measures of each animal across 8 sections (8 repeated measures for four WT LNASCB-, two R6/2 LNA-SCB-, and three R6/2 LNA-CTG-injected animals). Representative images of DARPP-32 staining in each condition are shown. Scale bar: $500 \mu \mathrm{m}$.
The pooled sRNAs transfected into cells contained enriched amounts of sCAG (Supplemental Figure 14). However, in the present study sCAG molecules were moderately increased in the striatum of R6/2 mice (1.22 fold change, $P=0.09$ ) (Supplemental Figure 14), compared with the originally reported 2-fold increase (9), which may be related with the shorter CAG repeat length in the $\mathrm{R} 6 / 2$ mouse model used in this study. It remains to be determined whether the beneficial effect of LNA-CTG is the consequence of it blocking the production and activity of SCAG and/or other sRNAs.

Decreased transcription of rRNA leading to nucleolar stress is another detrimental mechanism directly induced by RNA with expanded CAG repeats, including in $\mathrm{R} 6 / 2$ mice $(5,28)$. In agreement with this, the levels of $R n 45 s$ ( $45 \mathrm{~S}$ pre-ribosomal
RNA) were significantly decreased in R6/2 mice compared with levels in WT mice at 16 weeks of age (Supplemental Figure 15). At this time point (5 weeks after injection), R6/2 mice receiving LNA-CTG showed strong increases in Rn45s levels compared with those in R6/2 and WT mice injected with LNA-SCB (Supplemental Figure 15). No decreased levels of Rn45s or modifications upon LNA-CTG treatment were detected 5 days after injection, suggesting that $R n 45 s$ expression recovery could contribute to improvement at later time points.

Overall, these data suggest that detrimental mechanisms directly driven by expanded CAG repeat RNA are strongly reversed by LNA-CTG injection into the striatum of R6/2 mice and that this reversal contributes to LNA-CTG-mediated motor improvement. 
A
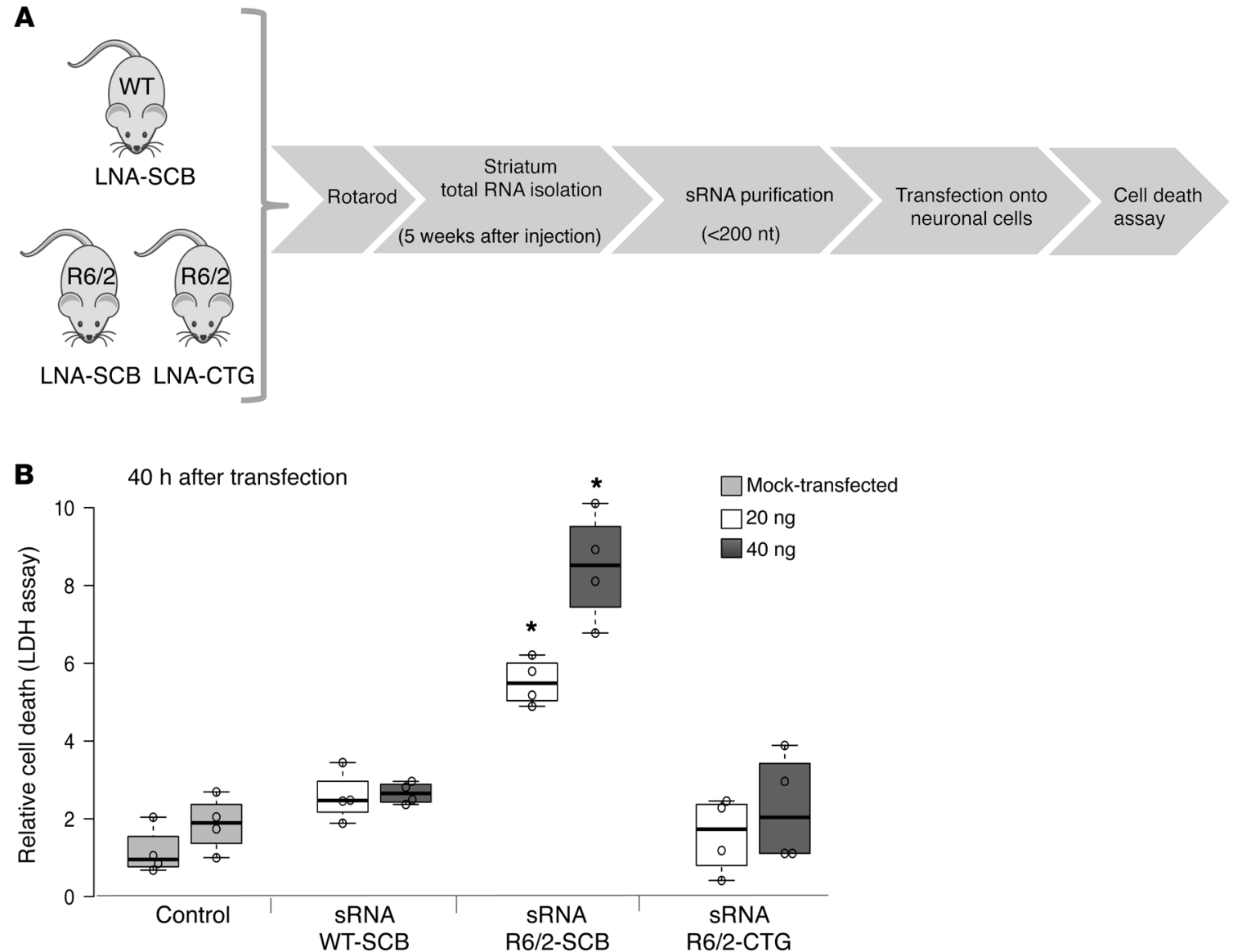

Figure 5. LNA-CTGs reverse the toxicity produced by sRNAs extracted from the striatum of R6/2 mice. (A) Workflow to evaluate the toxic effect of sRNAs isolated from mice injected with LNA-SCB or LNA-CTC, after motor performance evaluation. (B) Differentiated SH-SY5Y cells were transfected with sRNAs (20 ng or $40 \mathrm{ng}$ per well) from WT or R6/2 mice injected with LNA-SCB or from R6/2 mice injected with LNA-CTC, and cell death was evaluated 40 hours later using the lactate dehydrogenase (LDH) assay. Box plot shows relative cell death in each condition referred to a control mock-transfected sample with a value of $1 .{ }^{*} P<0.05$, by Kruskal-Wallis test, followed by a Mann-Whitney $U$ test with Bonferroni's correction as a post-hoc test $(n=4)$.

\section{Discussion}

Inhibition of mutant HTT expression has been proposed as a major therapeutic strategy in treating HD. Our efforts have surprisingly shown that blocking the activity of expanded CAG repeats in HTT-e1 transcripts without modifying HTT RNA or protein levels is sufficient to reverse motor symptoms in an HD mouse model.

Several types of LNA-ASOs complementary to the CAG repeats have been used for allele-selective inhibition in HD human fibroblasts at the protein level, with virtually no effect on HTT mRNA $(16-18,29)$. These studies showed that the configuration of the LNA-ASO largely affects the efficiency of protein inhibition. LNA-ASOs formed by 6 or 7 GTC repeats with $3^{\prime}$ and $5^{\prime}$ terminal guanines and LNA nucleotides at every third thymine residue strongly inhibited protein expression $(16,17)$. However, those consisting of CTG repeats were less efficient $(17,18)$, which agrees with our finding of a lack of effect of LNACTG on HTT protein levels.

We have demonstrated that the lack of effect of LNA-CTG on HTT protein expression levels is accompanied by the efficient binding of LNA-CTG to the full-length HTT transcript in human fibroblasts and $\mathrm{Htt}$-knockin mouse striatal neuronal cells. We have shown that LNA-CTG binding to the CAG repeats is a strong barri- er for retrotranscription as previously suggested (18); and the consequent lack of RT-PCR amplification of fragments spanning the CAG repeat permitted tracking of LNA-CTG binding to mutant HTT-e1 and other genes. The characterization of LNA-CTG dosage effects in HD fibroblasts indicated a preferential binding to the expanded allele with a 6-fold selectivity, which agrees with previous studies focusing on the effect of other ASOs on HTT protein expression $(16,17,29,30)$.

The therapeutic potential of HTT expression inhibition has been preclinically tested in rodent models by using a non-alleleselective approach based on virally expressed small hairpin RNAs (31-33), siRNAs (34), and ASOs (35). In these studies, a significant decrease in HTT protein levels reduced neuropathological signs, ameliorated symptoms, and prolonged the survival of HD mice. In addition, allele-selective phosphorodiamidate morpholino ASOs targeting the CAG repeat have recently shown antidepressant activity in a mouse model of HD that was linked to decreased levels of mutant HTT protein expression (36). In contrast, our results using LNA-ASOs complementary to the CAG repeats suggest that inhibition of mutant HTT-e1 protein expression is not necessary for reversing motor deficits in R6/2 mice or blocking toxicity in a neuronal cell model. These data, along with the lack of effect of 
morpholino ASOs on HTT mRNA levels (36), bring into question whether behavioral improvement is caused by protein inhibition and/or blockage of expanded CAG repeats at the RNA level.

In this study, we used an R6/2 HD mouse model featuring 90 CAG repeats in the transgene, instead of the 150 CAG repeats originally described (37). The shorter CAG-repeat length translated into a less aggressive phenotype, with symptoms starting around week 11, the earliest symptomatic time point chosen for administration of LNA-CTG. Two consecutive intrastriatal injections of LNA-CTG ASOs substantially improved molecular pathology and motor symptoms as early as 1 week after administration, effects that lasted at least 5 weeks. Improvement in motor symptoms correlated with sustained LNA-CTG binding to expanded CAG repeats, which agrees with the high biological stability of LNAASOs with phosphorothioate-modified backbones (38). Whether significant recovery is feasible in older animals, in which motor deficits are more pronounced, remains to be determined.

LNA-CTG facilitated the partial recovery of protein levels that were strongly decreased in R6/2 mice. Changes in the expression of these proteins may reflect the rapid neuroprotective effect of LNA-CTG and preservation of striatal neuron function, consistent with LNA-CTG-mediated blockage of mutant HTT RNA toxicity in a neuronal cell model and with the striking amelioration of motor symptoms we observed in vivo.

One of the main drawbacks to targeting the CAG repeats as a therapeutic strategy in HD and other polyQ disorders is the lack of selectivity. Although we demonstrate a preferential binding to expanded CAG repeats, LNA-CTGs similarly bound to other genes with shorter, normal CAG repeats. However, the lack of a noticeable effect of LNA-CTGs on the motor performance of WT mice and on the expression levels of the targeted genes suggests that off-target binding to endogenous genes does not attenuate normal functionality.

Our data show that LNA-CTGs, at the concentrations used in R6/2 mice and in different cell models, strongly bind to the CAG repeats in $H T T-e 1$, without affecting transcript and protein levels. Thus, we propose that the beneficial activity of LNA-CTG in vivo may be due to its blocking the activity of the CAG repeat expansion in HTT-e1 RNA. In the present study, evidence suggests that LNA-CTGs reverse deleterious mechanisms directly dependent on an RNA toxic gain of function. Expanded CAG repeats form a hairpin-like secondary structure that causes RNA toxicity through diverse mechanisms involving aberrant interactions with proteins and consequent altered functions (12). These include dicer and nucleolin (NCL), which underlie deleterious sRNA expression profiles $(9,27)$ and decreased $R n 45 s$ expression levels mediating nucleolar stress $(5,28)$, respectively.

LNA-CTG binding to the CAG repeats may disrupt sequestration and alter the activity of dicer and NCL, leading to the reversal of sRNA toxicity and recovery of Rn45s levels in R6/2 mice. Supporting this, a similar mechanism has been recently shown for LNA-ASOs targeting expanded CUG repeats in DMPK transcripts, which prevented muscleblind-like splicing regulator 1 (MBNL1) sequestration and corrected alternative splicing abnormalities (39). Since MBNL1 also binds to expanded CAG repeats (7), it remains to be determined whether analogous corrections in alternative splicing patterns occur in R6/2 mice injected with LNA-CTG, which could underlie motor improvement.
Repeat-associated non-ATG (RAN) translation is another mechanism dependent on expanded CAG RNA hairpin-like structures that may contribute to HD pathogenesis (40). The possibility that LNA-CTG disrupts the biogenesis of RAN translation products needs further investigation.

LNA-CTG-mediated reversal of sRNA toxicity and recovery of $R n 45 s$ transcript levels suggest that these mechanisms underlie motor dysfunction in R6/2 mice. Accurate molecular and behavioral longitudinal studies in vivo, correlating the appearance of motor symptoms with the detection of the above-mentioned deleterious outcomes in the presence and absence of LNA-CTG, will help to unravel the relevance of each pathogenic mechanism.

In summary, our data strongly suggest that the beneficial effects of LNA-CTG depend, at least in part, on the blockage of direct RNA detrimental effects, which has important mechanistic implications in HD pathogenesis. Whether motor recovery depends on additional indirect effects of LNA-CTG on HTT downstream targets and pathogenic pathways remains to be resolved. Using LNA-CTGs could help to elucidate the highly complex details of HD pathogenesis involving both RNA- and protein-dependent mechanisms. Finally, since targeting CAG repeats could be a valuable strategy for other CAG/polyglutamine disorders, the results presented here could help to establish a general approach to treating several rare neurodegenerative disorders for which there is no currently cure.

\section{Methods}

\section{Cell lines and transfection}

Primary HD fibroblasts. Fibroblasts were obtained from Coriell Institute for Medical Research. Cells were maintained at $37^{\circ} \mathrm{C}$ and $5 \% \mathrm{CO}_{2}$ in Eagle MEM (Gibco, Life Technologies, Thermo Fisher Scientific) supplemented with $12.5 \%$ heat-inactivated FBS (Sigma-Aldrich), 2 $\mathrm{mM}$ L-glutamine, 100 units $/ \mathrm{ml}$ penicillin and $100 \mathrm{mg} / \mathrm{ml}$ streptomycin (Gibco, Thermo Fisher Scientific), and 50\% DMEM (Sigma-Aldrich). Fibroblasts were maintained for a maximum of 20 passages. We used GM04208 and GM04281 cell lines with mutant alleles carrying 44_CAG or 68_CAG repeats.

SH-SY5Y neuronal cells. SH-SY5Y neuroblastoma cells (catalog 94030304; Sigma-Aldrich) were grown in DMEM (Invitrogen, Thermo Fisher Scientific) supplemented with $10 \%$ inactivated FBS, and 100 units/ml penicillin and $100 \mathrm{mg} / \mathrm{ml}$ streptomycin (Gibco, Thermo Fisher Scientific). SH-SY5Y cells were differentiated with $10 \mathrm{mM}$ retinoic acid (Sigma-Aldrich) added to the growing media, for 4 days. Media were removed and replaced by fresh growth media containing $80 \mathrm{nM} 12-\mathrm{O}$-tetradecanoylphorbol-13-acetate (Sigma-Aldrich) for 5 additional days.

Knockin striatal cells. Conditionally immortalized WT (STHdh ${ }^{\left.\mathrm{Q} / \mathrm{Q}^{7}\right)}$ and mutant (STHdh ${ }^{\mathrm{Q11} / \mathrm{Q}^{111}}$ ) striatal neuronal progenitor cell lines expressing endogenous levels of normal and mutant $\mathrm{Htt}$ with 7 and 111 glutamines, respectively, have been described elsewhere (19). Cells were a gift of M.E. MacDonald (Massachusetts General Hospital, Boston, Massachusetts, USA). Cells were grown at $37^{\circ} \mathrm{C}$ in DMEM (Gibco, Thermo Fisher Scientific) supplemented with $10 \%$ FBS, $1 \%$ nonessential amino acids, $2 \mathrm{mM} \mathrm{L-glutamine,} \mathrm{and} 400 \mu \mathrm{g} /$ ml G418 (Geneticin; Invitrogen, Thermo Fisher Scientific).

Cell transfections were performed using Lipofectamine 2000 (Invitrogen, Thermo Fisher Scientific) according to the manufacturer's 
instructions and at a cell confluence of $60 \%$. Vectors expressing WT or mutant HTT-e1 (9) were transfected using concentrations between 0.6 $\mathrm{ng} / \mu \mathrm{l}$ and $0.3 \mathrm{ng} / \mu \mathrm{l}$. sRNAs were transfected in 2 different amounts (20 and $40 \mathrm{nmol}$ per well). LNA-ASOs were transfected at the dosages indicated in the figure legends. The LNA-ASO complementary to the CAG repeat (LNA-CTG) consisted of a 20-nt length oligonucleotide, CTGCTGCTGCTGCTGCTGCT, with LNA located every third T and a phosphorothioate-modified backbone. LNA-CTG and the control scrambled LNA-modified sequence (LNA-SCB) 5'-GTGTAACACGTCTATACGCCCA-3' were obtained from Exiqon. Lipofectamine alone was used for control (mock-transfected) cells.

\section{HD mouse model}

For this study, we used male WT and R6/2 transgenic mice (B6CBA background) expressing a human mutant $H T T$ exon 1 with 90 CAG repeats instead of the 150 repeats originally described (37). Original R6/2 mice were purchased from The Jackson Laboratory. Colony maintenance resulted in a decrease of the CAG repeat length, in agreement with CAG repeat instability as previously described by other groups (41-43). Mouse genotype was determined as previously described (44). CAG repeat length was determined by PCR amplification of the repeat using HD1 and HD2 fluorescently labeled primers (45) and subsequent size determination in an ABI 3100 analyzer (Applied Biosystems). These results were further validated by Laragen Inc. All mice were housed together in numerical birth order in groups of mixed genotypes, and data were recorded for analysis by microchip mouse number. The animals were housed with ad libitum access to food and water in a colony room kept at $19^{\circ} \mathrm{C}$ to $22^{\circ} \mathrm{C}$ and $40 \%-60 \%$ humidity, under a 12-hour light/12-hour dark cycle.

\section{Behavioral analysis}

Rotarod. Motor coordination and balance were evaluated on the rotarod apparatus at distinct rotations per minute (rpm), as described elsewhere (46). Animals were trained at a constant speed ( $24 \mathrm{rpm}$ ) for 60 seconds at 4 or 8 weeks of age. We performed 2 trials per day for 3 consecutive days, and the number of falls during 60 seconds was recorded. No differences between groups were detected during this period. After training, animals were evaluated once a week at 16, 24, and $32 \mathrm{rpm}$, and the number of falls in a total of 60 seconds was recorded. The animals were put on the rotarod several times until the addition of the latency to falloff reached a total of 60 seconds. The curves representing the behavioral pattern were compared, and the percentage of motor coordination function impairment was calculated as described elsewhere (47).

Hanging wire. Neuromuscular abnormalities were analyzed by the hanging wire test as described elsewhere (46), beginning 1 week after surgery. A standard wire cage lid was used. To test balance and grip strength, mice were placed on top of a wire cage lid. The lid was shaken slightly to cause the mouse to grip the wires and then turned upside down for 60 seconds. The latency to fall was recorded.

\section{Intrastriatal injection}

Eleven-week-old WT and R6/2 mice were deeply anesthetized with pentobarbital $(50 \mathrm{mg} / \mathrm{kg})$ and placed in a stereotaxic apparatus for a bilateral injection into the striatum. Then, $2 \mu \mathrm{l}$ of $25 \mu \mathrm{M}$ LNA-SCB or LNA-CTG (final amount, 0.05 nmols) was injected at 2 different coordinates relative to bregma: (a) anteroposterior (AP) $+0.3 \mathrm{~mm}$; mediolateral (ML) $\pm 2 \mathrm{~mm}$; and (b) $\mathrm{AP}+0.8 \mathrm{~mm} ; \mathrm{ML} \pm 1.8 \mathrm{~mm}$; in both cases at $2.6 \mathrm{~mm}$ below the dural surface with the incisor bar at $3 \mathrm{~mm}$ above the interaural line. These injections were given twice per mouse, separated by 3 days. A 10- $\mu$ l Hamilton microliter syringe was used for the injections at an infusion rate of $0.25 \mu \mathrm{l} /$ minute, and the needle was left in place for 7 minutes to ensure complete diffusion and then slowly retracted from the brain. Mice were allowed to recover for 1 week before undergoing the rotarod and hanging wire tests.

Mice were killed by decapitation 2, 10, or 32 days after intrastriatal injection of LNA-SCB or LNA-CTG. Immediately after decapitation, brains were removed and halved sagittally. The hemispheres were processed for histology and protein or RNA extraction.

\section{Total protein extraction and Western blot analysis}

Cells were washed once with PBS and then incubated with lysis buffer at the indicated post-transfection time points (Figure 1, B and C, Figure 5B, Supplemental Figure 2, Supplemental Figure 3B, Supplemental Figure 4, and Supplemental Figure 10). Cells and tissues were homogenized in lysis buffer containing 1\% Triton X-100, $50 \mathrm{mM}$ Tris- $\mathrm{HCl}$ (pH 7.5), $10 \mathrm{mM}$ EGTA, $150 \mathrm{mM} \mathrm{NaCl}$, protease inhibitors (2 mM PMSF, $10 \mu \mathrm{g} / \mu \mathrm{l}$ aprotinin, $1 \mu \mathrm{g} / \mathrm{l}$ leupeptin), and phosphatase inhibitors $\left(2 \mathrm{mM} \mathrm{Na}_{3} \mathrm{VO}_{4}, 100 \mathrm{mM} \mathrm{NaF}\right)$ and centrifuged at $16,100 \times g$ for 20 minutes at $4^{\circ} \mathrm{C}$. The supernatants were collected, and protein concentration was measured using the DC Protein Assay Kit (BioRad). Western blot analysis was performed as previously described (48). The following primary antibodies were used: anti-HTT (1:1,000, catalog MAB2166, clone 1HU-4C8; 1:2,000, catalog MAB5492); anti-mutant HTT (1:1,000, catalog MAB5492, clone EM48); and antipolyQ (1:1,000, catalog MAB1574, clone 5TF1-1C2) from EMD Millipore; anti-DARPP32 (1:1,000, catalog 611520, clone 15/DARPP-32; BD Biosciences); anti-ENK (1:1,000; a gift of Dr. Dickson, Department of Emergency Medicine, University of Iowa, Iowa City, USA; ref. 49); anti-PHLPP1 (1:500, catalog 10007191; Cayman Chemical); anti-STEP (1:1,000, catalog sc-23892, clone 23E5; Santa Cruz Biotechnology Inc.); anti-PSD-95/SAP-90 (1:1,000, catalog MA1-046, clone 7E3-1B8; Thermo Fisher Scientific); anti-MAML2 (1:1,000, catalog 6988, clone D41E6; Cell Signaling Technology); anti-MEF2A (1:1,000, catalog 9736; Cell Signaling Technology); anti-SATB1 (1:1,000, catalog ab7004; Abcam); and anti-GR/NR3C1 (1:1,000, catalog NB300-731, clone BuGR2; Novus Biologicals). The loading control was performed by reprobing the membranes with an anti- $\alpha-$ tubulin antibody (1:50,000, catalog T9026, clone DM1A; SigmaAldrich) for 20 minutes at room temperature. Then, membranes were washed with Tris-buffered saline containing 0.1\% Tween-20 (TBS-T), incubated for 1 hour at room temperature with the corresponding HRP-conjugated antibody (1:2,000, anti-mouse IgG [H+L], catalog W402B and anti-rabbit IgG [H+L], W401B; both from Promega), and washed again with TBS-T. Immunoreactive bands were visualized using the Western Blotting Luminol Reagent (Santa Cruz Biotechnology Inc.) and quantified by a computer-assisted densitometer (Gel-Pro Analyzer, version 4; Media Cybernetics).

\section{IHC and morphological analysis in mouse brains}

Cerebral hemispheres were fixed by immersion in $4 \%$ paraformaldehyde solution in $0.1 \mathrm{M}$ sodium phosphate, $\mathrm{pH} 7.2$, for 3 days. Tissues were then cryoprotected by immersion for 24 hours in $10 \%$, $20 \%$, and $30 \%$ sucrose in PBS with $0.02 \%$ sodium azide and frozen in dry ice-cooled 2-methylbutane. Serial coronal sections $(30-\mu \mathrm{m}$ 
thick) were cut on a cryostat. For IHC, sections (processed as free floating) were washed 3 times in PBS, incubated for 30 minutes in a $50-\mathrm{mM} \mathrm{NH}_{4} \mathrm{Cl}$ solution, permeabilized, and blocked by incubating for 1 hour at room temperature with PBS containing 0.5\% Triton $\mathrm{X}-100,3 \%$ normal horse serum (Pierce Biotechnology, Thermo Fisher Scientific), and 0.2\% BSA. After 3 washes (PBS with 0.5\% Triton $\mathrm{X}-100)$, brain slices were incubated with anti-DARPP-32 antibodies (1:1,000; BD Biosciences) or anti-mutant HTT (1:150, EM48; EMD Millipore) overnight at $4^{\circ} \mathrm{C}$. After primary antibody incubation, slices were washed 3 times (PBS with 0.5\% Triton X-100) and then incubated for 2 hours at room temperature with the corresponding fluorescent secondary antibodies: Cy2 goat anti-guinea pig (1:500) and Cy3 goat anti-mouse (1:200) (both from Jackson ImmunoResearch). No signal was detected in controls incubated in the absence of primary antibodies. For nuclear staining, sections were incubated for 10 minutes at room temperature with Hoechst 33258 (1:4,000; Invitrogen, Thermo Fisher Scientific). DARPP-32 immunofluorescence was analyzed by confocal microscopy in coronal sections evenly spaced at $240 \mu \mathrm{m}$ apart comprising rostral, middle, and caudal levels of the striatum ( $1.54 \mathrm{~mm}$ to $-0.7 \mathrm{~mm}$ from bregma) as described elsewhere (50).

\section{RNA foci detection by FISH in HD fibroblasts}

RNA FISH was performed using a DNA/LNA probe (CTG)6-CA labeled at the $5^{\prime}$ end with $\mathrm{Cy} 3$ and following previously described procedures (6). Slides were mounted in Fluoroshield with DAPI medium (Sigma-Aldrich). Control experiments included RNAse A treatment $\left(0.01 \mu \mathrm{g} / \mathrm{ml}\right.$ for 1.5 hours at $\left.37^{\circ} \mathrm{C}\right)$ prior to FISH staining. RNA foci were not detected in these conditions (data not shown). Cells were visualized using a Leica Confocal TCS SPE microscope.

\section{Cell toxicity assays}

Lactate dehydrogenase (LDH) released from dying cells was determined using the LDH assay (Cytotox 96; Promega) according to the manufacturer's protocol. Absorbance was recorded at $490 \mathrm{~nm}$, at different time points after transfection (see figure legends). LDH determinations were performed in quadruplicate.

\section{RNA isolation}

The mouse dissected brain areas were placed immediately in QIAzol solution (QIAGEN), followed by RNA extraction with the miRNeasy kit (QIAGEN) as indicated by the manufacturer. RNA extraction from the neuronal cells or primary fibroblasts was also performed with miRNeasy (QIAGEN) following the manufacturer's instructions. Determinations of RNA quality and quantity were made with a 2100 Bioanalyzer (Agilent Technologies) and an ND-1000 spectrophotometer (Thermo Fisher Scientific), respectively. All RNA samples showed an RNA integrity number of 8 or higher.

sRNA fractions from DNAse I-treated total RNA were purified with the RNA Clean \& Concentrator-5 Kit (Zymo Research) according to the manufacturer's instructions.

Transcription of HTT-e1 and retrotranscription in the presence of LNA-ASOs A plasmid containing a human $H T T-e 1$ with 23 CAG repeats (9) was used PCR amplification of the HTT-e1 sequence and the upstream T7 promoter. The PCR product was used as a template to synthesize a pure HTT-e1 RNA using the MAXIscript T7 Transcription Kit (Invitrogen,
Thermo Fisher Scientific). HTT-e1 RNA was used to evaluate retrotranscription performance in the presence of bound LNA-ASOs. HTT-e1 RNA was first incubated with either LNA-CTG or LNA-SCB at a molar ratio of 1:5 (HTT-e1/LNA-ASOs) at $37^{\circ} \mathrm{C}$ for 1 hour. Retrotranscription was then performed with the SuperScript III First-Strand Synthesis System (Invitrogen), using a sequence-specific primer (see Supplemental Table 1 for primer descriptions). Retrotranscription products were resolved by denaturing polyacrylamide gel electrophoresis.

\section{Microarray hybridization and analysis}

Total RNA (100 ng) was labeled using the Low Input Quick Amp Labeling Kit (Agilent Technologies) following the manufacturer's instructions. Briefly, mRNA was reverse transcribed in the presence of T7-oligo-dT primer to produce cDNA, which was then in vitro transcribed with T7 RNA polymerase in the presence of Cy3-CTP to produce labeled cRNA. The labeled cRNA was hybridized to the Agilent SurePrint G3 Mouse GE $8 \times 60 \mathrm{~K}$ Microarray according to the manufacturer's protocol. The arrays were washed and scanned on an Agilent G2565CA microarray scanner at 100\% photomultiplier tube (PMT) and $3 \mu \mathrm{m}$ resolution. Intensity data were extracted using the Agilent Feature Extraction software.

Raw data were taken from the Feature Extraction output files and corrected for background noise using the normexp method (51) (Gene Expression Omnibus [GEO] accession number GSE85126). To assure comparability across samples, we used quantile normalization (52). Differential expression analysis was carried out on noncontrol probes with an empirical Bayesian approach on linear models (limma) (53). Results were corrected for multiple testing according to the FDR method (54). All statistical analyses were performed using Bioconductor software (http://www.bioconductor.org/) in the R statistical environment (http://cran.r-project.org/) (55).

\section{Semiquantitative RT-PCR of human HTT}

Equal amounts of RNA samples $(1 \mu \mathrm{g})$ were treated with DNAse I (Ambion, Thermo Fisher Scientific), and 500 ng was reverse transcribed with the Transcriptor First Strand cDNA Synthesis Kit (Roche) according to the manufacturer's protocol, and PCR was performed using the FastStart Taq DNA Polymerase, dNTPack (Roche). sCAGs were determined as previously described (9). Briefly, RNA samples were polyadenylated and annealed with a polyT adapter prior to retrotranscription. Then, PCR amplification was performed using primers recognizing the adapter and sCAG.

PCR products were resolved by electrophoresis in $2 \%$ agarose gels and quantified using ImageJ software (NIH). The primers and PCR conditions used for HTT, $\beta$-actin, Gdx, and GFP cDNA (Figure 1, Figure 2, and Supplemental Figures 1-3, 9, and 10) are indicated in Supplemental Table 2 .

\section{qPCR}

Total RNA from mouse striatum was treated with DNAse I (Ambion, Thermo Fisher Scientific) and a retrotranscribed using Transcriptor First Strand cDNA Synthesis Kit (Roche) following the manufacturer's instructions. cDNA was mixed with LightCycler 480 SYBR Green I and amplification performed in a LightCycler 480 Real-Time PCR System (Roche). For each gene, samples were analyzed in the same RT-PCR experiment and run in quadruplicate. Relative quantification (RQ) was calculated with the $2 \Delta \Delta \mathrm{Ct}$ method (56) using Hprt or 
$T b p$ as reference genes. RQ was calculated to compare all expression values normalized to the reference genes in WT animals injected with LNA-SCB, WT animals injected with LNA-CTG, R6/2 mice treated with LNA-SCB, and R6/2 mice treated with LNA-CTG (Supplemental Figures 11-13 and Table 1). The primers used for qPCR and amplification efficiencies and amplification conditions are listed in Supplemental Table 3.

\section{Statistics}

Data are represented in box plots or expressed as the mean \pm SEM (detailed information about the number of replicates in each condition or group is provided in the figure legends). Statistical analyses were performed using 2-way ANOVA with repeated measures (Figure 2, Figure 4B, and Supplemental Figure 5B); a nonparametric Mann-Whitney $U$ test (Figure 1A, Figure 2D, Supplemental Figure 6, Supplemental Figure 10A, and Supplemental Figure 14A); or the Kruskal-Wallis test when more than 2 conditions were compared, followed by a Mann-Whitney $U$ test with Bonferroni's post-hoc correction (Figure 2C, Figure 4A, Figure 5B, Supplemental Figure 12, and Supplemental Figure 4B). A $P$ value of less than 0.05 was considered statistically significant. Statistical significance of RQs in qPCR (Figure 3 and Supplemental Figures 11-13 and 15) were obtained from a linear mixed-effects model (57), which accounts for the different sources of variation derived from the experimental design (58).

\section{Study approval}

All procedures were performed in compliance with the NIH guidelines for the care and use of laboratory animals and approved by the IACUCs of the Universitat de Barcelona (99/01) and the Generalitat de Catalunya (99/1094).

\section{Author contributions}

$\mathrm{EM}, \mathrm{XE}$, and EPN designed the research studies. LR, MBC, JCM, AG, and RAV designed and performed experiments. GM, BK, MTZA, ZA, and VV performed experiments. LR, MBC, JCM, AG, RAV, GM, BK, MTZA, ZA, VV, EPN, and EM analyzed and interpreted the data. All authors made intellectual contributions to the experimental design and discussion. EM and EPN wrote the manuscript.

\section{Acknowledgments}

This work was supported by the Spanish government through the Plan Nacional de I+D+I and cofunded by grants from the Instituto de Salud Carlos III (ISCIII) - Subdirección General de Evaluación and the Fondo Europeo de Desarrollo Regional (FEDER) (project PI11/02036, to EM, and PI13/01250, to EPN); the Spanish Ministerio de Economía y Competitividad (MINECO) (SAF2008-00357 and SAF2013-49108-R, to XE, and SAF2014-60551-R: iRPaD, to EM); and the Generalitat de Catalunya, Departament Economia i Coneixement, Secretaria Universitats i Recerca (AGAUR 2014 SGR-1138, to XE). We acknowledge support of the Spanish Ministry of Economy and Competitiveness, 'Centro de Excelencia Severo Ochoa 2013-2017.' We thank the staff of the Genomics Unit at the CRG for microarray analysis; A. López and M.T. Muñoz for their technical support; S. Bonnal for her help with the in vitro retrotranscription experiments; G. Escaramís for her help with statistical analysis; and V. Raker for editing of the manuscript.

Address correspondence to: Eulàlia Martí or Xavier Estivill, Dr. Aiguader 88, E-08003 Barcelona, Catalonia, Spain. Phone: 34.933.160.201; E-mail: eulalia.marti@crg.cat (E. Martí). Phone: 34.933.160.138; E-mail: xavier.estivill@crg.cat (X. Estivill).
1. A novel gene containing a trinucleotide repeat that is expanded unstable on Huntington's disease chromosomes. The Huntington's Disease Collaborative Research Group. Cell. 1993;72(6):971-983.

2. Cattaneo E, Zuccato C, Tartari M. Normal huntingtin function: an alternative approach to Huntington's disease. Nat Rev Neurosci. 2005;6(12):919-930.

3. Takahashi T, Katada S, Onodera O. Polyglutamine diseases: Where does toxicity come from? What is toxicity? Where are we going? J Mol Cell Biol. 2010;2(4):180-191.

4. Labbadia J, Morimoto RI. Huntington's disease: underlying molecular mechanisms and emerging concepts. Trends Biochem Sci. 2013;38(8):378-385.

5. Tsoi H, Lau TC, Tsang SY, Lau KF, Chan HY. CAG expansion induces nucleolar stress in polyglutamine diseases. Proc Natl Acad Sci U S A. 2012;109(33):13428-13433.

6. de Mezer M, Wojciechowska M, Napierala M, Sobczak K, Krzyzosiak WJ. Mutant CAG repeats of Huntingtin transcript fold into hairpins, form nuclear foci and are targets for RNA interference. Nucleic Acids Res. 2011;39(9):3852-3863.

7. Mykowska A, Sobczak K, Wojciechowska M, Kozlowski P, Krzyzosiak WJ. CAG repeats mimic CUG repeats in the misregulation of alternative splicing. Nucleic Acids Res. 2011;39(20):8938-8951.

8. Galka-Marciniak P, Urbanek MO, Krzyzosiak WJ.
Triplet repeats in transcripts: structural insights into RNA toxicity. Biol Chem. 2012;393(11):1299-1315.

9. Bañez-Coronel M, et al. A pathogenic mechanism in Huntington's disease involves small CAG-repeated RNAs with neurotoxic activity. PLoS Genet. 2012;8(2):e1002481.

10. Jasinska A, et al. Structures of trinucleotide repeats in human transcripts and their functional implications. Nucleic Acids Res. 2003;31(19):5463-5468.

11. Krzyzosiak WJ, Sobczak K, Wojciechowska M, Fiszer A, Mykowska A, Kozlowski P. Triplet repeat RNA structure and its role as pathogenic agent and therapeutic target. Nucleic Acids Res. 2012;40(1):11-26.

12. Nalavade R, Griesche N, Ryan DP, Hildebrand S, Krauss S. Mechanisms of RNA-induced toxicity in CAG repeat disorders. Cell Death Dis. 2013;4:e752.

13. Zu T, et al. Non-ATG-initiated translation directed by microsatellite expansions. Proc Natl Acad Sci U S A. 2011;108(1):260-265.

14. Matsui M, Corey DR. Allele-selective inhibition of trinucleotide repeat genes. Drug Discov Today. 2012;17(9-10):443-450.

15. Fiszer A, Krzyzosiak WJ. Oligonucleotide-based strategies to combat polyglutamine diseases. Nucleic Acids Res. 2014;42(11):6787-6810.

16. Hu J, Matsui M, Corey DR. Allele-selective inhibition of mutant huntingtin by peptide nucleic acid-peptide conjugates, locked nucleic acid, and small interfering RNA. Ann N Y Acad Sci. 2009;1175:24-31.

17. Gagnon KT, et al. Allele-selective inhibition of mutant huntingtin expression with antisense oligonucleotides targeting the expanded CAG repeat. Biochemistry. 2010;49(47):10166-10178.

18. Fiszer A, et al. An evaluation of oligonucleotide-based therapeutic strategies for polyQ diseases. BMC Mol Biol. 2012;13:6.

19. Gines S, Ivanova E, Seong IS, Saura CA, MacDonald ME. Enhanced Akt signaling is an early pro-survival response that reflects N-methyl-Daspartate receptor activation in Huntington's disease knock-in striatal cells. J Biol Chem. 2003;278(50):50514-50522.

20. Nucifora FC, et al. Interference by huntingtin and atrophin-1 with cbp-mediated transcription leading to cellular toxicity. Science. 2001;291(5512):2423-2428.

21. Luthi-Carter R, et al. Decreased expression of striatal signaling genes in a mouse model of Huntington's disease. Hum Mol Genet. 2000;9(9):1259-1271.

22. Sun Z, Del Mar N, Meade C, Goldowitz D, Reiner A. Differential changes in striatal projection neurons in R6/2 transgenic mice for Huntington's disease. Neurobiol Dis. 2002;11(3):369-385.

23. Torres-Peraza JF, Giralt A, García-Martínez JM, Pedrosa E, Canals JM, Alberch J. Disruption of 
striatal glutamatergic transmission induced by mutant huntingtin involves remodeling of both postsynaptic density and NMDA receptor signaling. Neurobiol Dis. 2008;29(3):409-421.

24. Saavedra A, et al. PH domain leucine-rich repeat protein phosphatase 1 contributes to maintain the activation of the PI3K/Akt pro-survival pathway in Huntington's disease striatum. Cell Death Differ. 2010;17(2):324-335.

25. Saavedra A, et al. Striatal-enriched protein tyrosine phosphatase expression and activity in Huntington's disease: a STEP in the resistance to excitotoxicity. J Neurosci. 2011;31(22):8150-8162.

26. Krol J, Fiszer A, Mykowska A, Sobczak K, de Mezer M, Krzyzosiak WJ. Ribonuclease dicer cleaves triplet repeat hairpins into shorter repeats that silence specific targets. Mol Cell. 2007;25(4):575-586.

27. Lawlor KT, et al. Double-stranded RNA is pathogenic in Drosophila models of expanded repeat neurodegenerative diseases. Hum Mol Genet. 2011;20(19):3757-3768.

28. Tsoi H, Chan HY. Expression of expanded CAG transcripts triggers nucleolar stress in Huntington's disease. Cerebellum. 2013;12(3):310-312.

29. Hu J, et al. Allele-specific silencing of mutant huntingtin and ataxin-3 genes by targeting expanded CAG repeats in mRNAs. Nat Biotechnol. 2009;27(5):478-484.

30. Gagnon KT, et al. Antisense and antigene inhibition of gene expression by cell-permeable oligonucleotide-oligospermine conjugates. J Am Chem Soc. 2011;133(22):8404-8407.

31. Boudreau RL, et al. Nonallele-specific silencing of mutant and wild-type huntingtin demonstrates therapeutic efficacy in Huntington's disease mice. Mol Ther. 2009;17(6):1053-1063.

32. Drouet $V$, et al. Sustained effects of nonallele-specific Huntingtin silencing. Ann Neurol. 2009;65(3):276-285.

33. Harper SQ, et al. RNA interference improves motor and neuropathological abnormalities in a Huntington's disease mouse model. Proc Natl Acad Sci U S A. 2005;102(16):5820-5825.

34. DiFiglia $M$, et al. Therapeutic silencing of mutant huntingtin with siRNA attenuates striatal and cortical neuropathology and behavioral deficits. Proc Natl Acad Sci U S A. 2007;104(43):17204-17209.

35. Kordasiewicz HB, et al. Sustained therapeutic reversal of Huntington's disease by transient repression of huntingtin synthesis. Neuron. 2012;74(6):1031-1044.

36. Sun X, et al. Phosphorodiamidate morpholino oligomers suppress mutant huntingtin expression and attenuate neurotoxicity. Hum Mol Genet. 2014;23(23):6302-6317.

37. Mangiarini L, et al. Exon 1 of the HD gene with an expanded CAG repeat is sufficient to cause a progressive neurological phenotype in transgenic mice. Cell. 1996;87(3):493-506.

38. Bennett CF, Swayze EE. RNA targeting therapeutics: molecular mechanisms of antisense oligonucleotides as a therapeutic platform. Annu Rev Pharmacol Toxicol. 2010;50:259-293.

39. Wojtkowiak-Szlachcic A, et al. Short antisense-locked nucleic acids (all-LNAs) correct alternative splicing abnormalities in myotonic dystrophy. Nucleic Acids Res. 2015;43(6):3318-3331.

40. Bañez-Coronel M, et al. RAN Translation in Huntington Disease. Neuron. 2015;88(4):667-677.

41. Dragatsis I, et al. CAG repeat lengths $\geq 335$ attenuate the phenotype in the R6/2 Huntington's disease transgenic mouse. Neurobiol Dis. 2009;33(3):315-330.

42. Møllersen L, Rowe AD, Larsen E, Rognes T, Klungland A. Continuous and periodic expansion of CAG repeats in Huntington's disease R6/1 mice. PLoS Genet. 2010;6(12):e1001242.

43. Morton AJ, et al. Paradoxical delay in the onset of disease caused by super-long CAG repeat expansions in R6/2 mice. Neurobiol Dis. 2009;33(3):331-341

44. Giralt A, Saavedra A, Carretón O, Xifró X, Alberch J, Pérez-Navarro E. Increased PKA signaling disrupts recognition memory and spatial memory: role in Huntington's disease. Hum Mol Genet. 2011;20(21):4232-4247.

45. The Huntington's Disease Collaborative Research Group. A novel gene containing a trinucleotide repeat that is expanded unstable on Huntington's disease chromosomes. Cell. 1993;72(6):971-983.

46. Giralt A, Carretón O, Lao-Peregrin C, Martín ED, Alberch J. Conditional BDNF release under pathological conditions improves Huntington's disease pathology by delaying neuronal dysfunction. Mol Neurodegener. 2011;6(1):71.

47. Ferrante RJ, et al. Therapeutic effects of coen- zyme Q10 and remacemide in transgenic mouse models of Huntington's disease. J Neurosci. 2002;22(5):1592-1599.

48. Rué L, López-Soop G, Gelpi E, Martínez-Vicente $\mathrm{M}$, Alberch J, Pérez-Navarro E. Brain region- and age-dependent dysregulation of p62 and NBR1 in a mouse model of Huntington's disease. Neurobiol Dis. 2013;52:219-228.

49. Watson JD, Varley JG, Tomlin SJ, Medbak S, Rees LH, Hinds CJ. Biochemical characterization of circulating Met-enkephalins in canine endotoxin shock. J Endocrinol.1986;111(2):329-334.

50. Brito V, Puigdellívol M, Giralt A, del Toro D, Alberch J, Ginés S. Imbalance of p75(NTR)/ TrkB protein expression in Huntington's disease: implication for neuroprotective therapies. Cell Death Dis. 2013;4:e595.

51. Ritchie ME, et al. A comparison of background correction methods for two-colour microarrays. Bioinformatics. 2007;23(20):2700-2707.

52. Bolstad BM, Irizarry RA, Astrand M, Speed TP. A comparison of normalization methods for high density oligonucleotide array data based on variance and bias. Bioinformatics. 2003;19(2):185-193.

53. Smyth GK. Linear models and empirical bayes methods for assessing differential expression in microarray experiments. Stat Appl Genet Mol Biol. 2004;3:Article3.

54. Benjamini Y, Hochberg Y. Controlling the false discovery rate: a practical and powerful approach to multiple testing. JR Stat Soc Series B Stat Methodol.1995;57(1), 289-300. http://www.jstor.org/ stable/2346101. Accessed September 29, 2016.

55. Gentleman RC, et al. Bioconductor: open software development for computational biology and bioinformatics. Genome Biol. 2004;5(10):R80.

56. Livak KJ, Schmittgen TD. Analysis of relative gene expression data using real-time quantitative PCR and the 2(-Delta Delta C(T)) Method. Methods. 2001;25(4):402-408.

57. Steibel JP, Poletto R, Coussens PM, Rosa GJ. A powerful and flexible linear mixed model framework for the analysis of relative quantification RT-PCR data. Genomics. 2009;94(2):146-152.

58. Miñones-Moyano E, et al. MicroRNA profiling of Parkinson's disease brains identifies early downregulation of miR-34b/c which modulate mitochondrial function. Hum Mol Genet. 2011;20(15):3067-3078. 